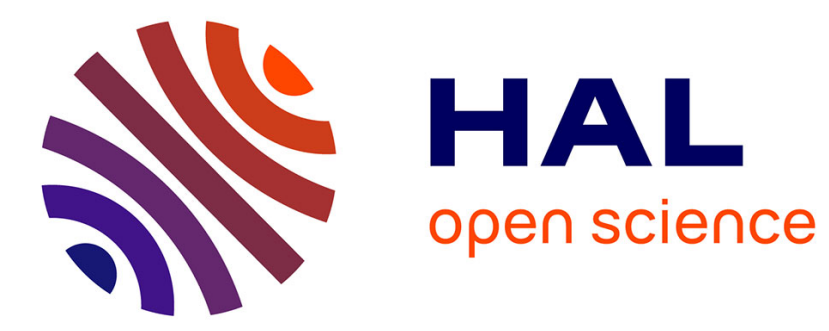

\title{
Wide Frequency Range Lumped Element Equivalent Circuit for HF Planar Transformer
}

Abdelhadi Besri, Xavier Margueron, Jean-Pierre Keradec, Benoît Delinchant

\section{To cite this version:}

Abdelhadi Besri, Xavier Margueron, Jean-Pierre Keradec, Benoît Delinchant. Wide Frequency Range Lumped Element Equivalent Circuit for HF Planar Transformer. 2008 IEEE 39th Annual PESC (Power Electronics Specialists Conference), Jun 2008, Rhodes, Greece. hal-00289675

\section{HAL Id: hal-00289675 https://hal.science/hal-00289675}

Submitted on 13 Feb 2009

HAL is a multi-disciplinary open access archive for the deposit and dissemination of scientific research documents, whether they are published or not. The documents may come from teaching and research institutions in France or abroad, or from public or private research centers.
L'archive ouverte pluridisciplinaire HAL, est destinée au dépôt et à la diffusion de documents scientifiques de niveau recherche, publiés ou non, émanant des établissements d'enseignement et de recherche français ou étrangers, des laboratoires publics ou privés. 


\title{
Wide Frequency Range Lumped Element Equivalent Circuit for HF Planar Transformer
}

\author{
A. Besri*, X. Margueron**, J-P. Keradec*, B. Delinchant* \\ * G2ELAB, UMR 5269- INPG -UJF -CNRS, ENSIEG, St Martin d'Hères Cedex, France \\ ** L2EP, Ecole Centrale de Lille, Cité Scientifique, Villeneuve d'Ascq Cedex, France
}

\begin{abstract}
Lumped element equivalent circuits that accurately represent the electrical behavior of H.F. nwinding transformers are now available. These circuits suit for time domain simulation but evaluation of their parameters relies on measurements. In order to avoid prototype manufacturing, engineers need to deduce same parameters from geometrical and physical data and, to carry out optimization, they prefer analytical approach to fem one. This paper shows how to do this for planar transformers which have simple geometry. Comparison of results with experimental data is given and analyzed.
\end{abstract}

\section{INTRODUCTION}

For a long time, representation of the electrical behavior of a H.F. transformer has been an unsolved problem. After several successive improvements, equivalent circuits able to do this accurately are now available, even for nwinding-components [1]. As long as transformers behave linearly, magnetic and capacitive couplings are correctly described, as well as losses, by lumped elements circuits.

Owing to the lumped elements nature of these circuits, they suit for time domain electronic simulation of the whole electronic circuit, with any circuit simulator like PSpice [2]. All the values of the components included in these circuits can be deduced from well chosen impedance measurements, according to methods which have been described elsewhere $[3,4,5]$.

Of course, using this way to know the parameters of the equivalent circuit supposes that a prototype has been built. Because recent technology prototypes are expensive and their realization is time consuming, engineers wish to reduce the number of such prototypes. With this in mind, methods to deduce the values of equivalent circuit parameters from the physical and geometrical description of the component are expected. In view of entering an optimization process, it is more desirable to do this by analytical calculation rather than by fem simulation.

Analytical studies related to this wide field are still in progress but, for simple shape planar transformers, needed theoretical material to do this is already available $[6,7]$. In this paper, we apply this theoretical work to deduce the equivalent circuit of such a planar transformer.

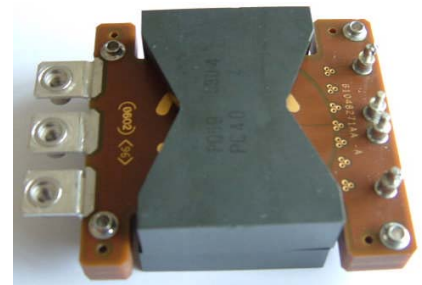

Fig. 1. A 9 kVA, 290 g, 3-winding planar transformer
Nowadays, planar transformers are widely used in H.F. converters because they are mechanically robust (due to the PCB structure of their windings), their electrical characteristics are reproducible (especially for capacitive aspects) and their thermal draining is efficient owing to their slim shape. Moreover, if carefully designed, they offer high power efficiency that make them very attractive for H.F. systems embedded in aircrafts (Fig. 1).

In section II- $A$, we precise geometrical assumptions on which our theoretical approach is based. Then (section II$B)$, we present this theory. According to it, each layer (conductive, insulating or magnetic) gone through inside the transformer is represented by an electrical circuit which, includes frequency dependant impedances. Equivalent circuit of the whole transformer is obtained by connecting all together these elementary circuits.

In section II- $C$, a transformation of this circuit that avoids presence of negative real part impedances is presented. Then, (section II- $D$ ) frequency dependant impedances are replaced by sets of lumped elements in small numbers in order to ease time domain simulations.

Section II- $E$ is dedicated to the capacitive aspects representation. The above mentioned model doesn't take this into account. For this reason, an independent and simple model is added.

Finally, in section III, an industrial transformer (Fig. 1) is studied. We catch this opportunity to explain how to use specifications of the ferrite core to account its losses, the effect of grinding and the introduction of an air gap. For this component, measurements are compared to model predictions. The fit is quite good and most of observed disagreements are explained. Some of these explanations are experimentally checked.

\section{THEORETICAL BASIS}

\section{A. Geometry of the Concerned Transformers}

A large part of planar transformer appears as a stack of different types of layers which are either conductive or insulating or, also, magnetic. In such a physical structure, energy exchanges between conductive layers intervene mainly through plane waves perpendicular to the layers.

Derivation of the theoretical results supposes that each layer of the transformer is a finite width, finite length, piece of an infinite plane layer. Moreover, it is assumed that all layers of the stack have the same widths and lengths and that they are all aligned face to face.

According to these assumptions, two extensions can be considered. First, a conductive layer can be made of several parallel and identical wires connected in series. In this case, because the current is the same in all strands, 
current distribution through the width of the layer is quite uniform and results are close to that of a homogeneous layer. Second, as long as curvature radius is much larger than layer thickness, curvature can be neglected. This is why some transformers having windings made of cylindrically wound foil can be studied this way.

\section{B. Equivalent Circuit Representing a Conductive Plate}

Let us consider (Fig. 2) a parallelepiped piece of a homogenous, isotropic material. We also assume that this material behaves linearly, so, for harmonic studies, its electrical properties are described by a few complex parameters (permittivity, permeability and conductivity) which can vary with frequency. Indeed, as shown by Maxwell-Ampere equation (1), when conductivity is not null, it gives a major contribution to the imaginary part of permittivity and, in every case, it can be grouped with that parameter, so the material is completely described, at each frequency, by only two complex constants: permittivity $\bar{\varepsilon}$ and permeability $\bar{\mu}$.

$$
\begin{aligned}
& \operatorname{rot} H=\sigma E+j \omega \varepsilon_{d} E=j \omega \bar{\varepsilon} E \\
& \text { if: } \bar{\varepsilon}=\varepsilon_{d}+\frac{\sigma}{j \omega}
\end{aligned}
$$

Now, have a look to the device shown in figure 2. This piece of an infinite conductive plate of thickness $a$, normal to $O z$, has a width $b$ and a length $c$. The plate is exposed to incident and reflected plane waves on both its faces. Magnetic field is, anywhere, parallel to $O y$. An electric power supply, which forces a current $J$ per unit of width and a voltage $V$ per unit of length, is also connected to this plate. This external circuit adds a uniform electric field $E_{s}$ inside the plate and it allows conduction current to flow inside. Attenuation of a plane wave going through a homogenous and isotropic infinite plate is a well-known problem. It is shown [8] that, while going from one plate side to the other, complex amplitude of magnetic field is multiplied by $A$, given by (2). The same attenuation holds for the reverse direction of propagation.

$$
A=e^{-j \varphi} \quad \text { with: } \varphi=\omega \sqrt{\bar{\mu} \bar{\varepsilon}} a
$$

Accounting for continuity of electric and magnetic fields on both surfaces, it becomes possible to express (3) total electric field on left and right sides $\left(E_{l}\right.$ and $\left.E_{r}\right)$ and electric field $E_{\mathrm{s}}$ in terms of total magnetic field on both sides $\left(H_{l}\right.$ and $\left.H_{r}\right)$ and current per unit of width $J$.

$$
\begin{aligned}
& E_{l}-E_{s}=Z_{c} \frac{1-A}{1+A} H_{l}+Z_{c} \frac{2 A}{1-A^{2}} J \\
& E_{r}-E_{s}=Z_{c} \frac{2 A}{1-A^{2}} J-Z_{c} \frac{1-A}{1+A} H_{r} \\
& \text { where: } Z_{c}=\sqrt{\frac{\bar{\mu}}{\bar{\varepsilon}}}
\end{aligned}
$$

This linear system, which connects three power sources (an electromagnetic field on each face and an electric power supply), behaves as a three port circuit. However, considering that total magnetic field on both faces and total current circulating inside the layer are linked by Ampere's theorem, the three inputs of this circuit are not independent so its representation is simpler than expected (Fig. 3).

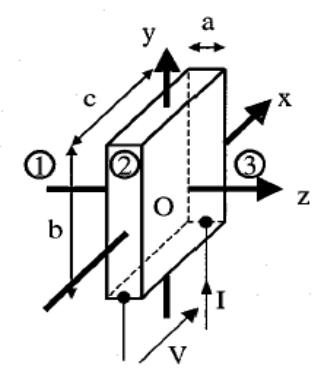

Fig. 2.Elementary device composing a planar transformer

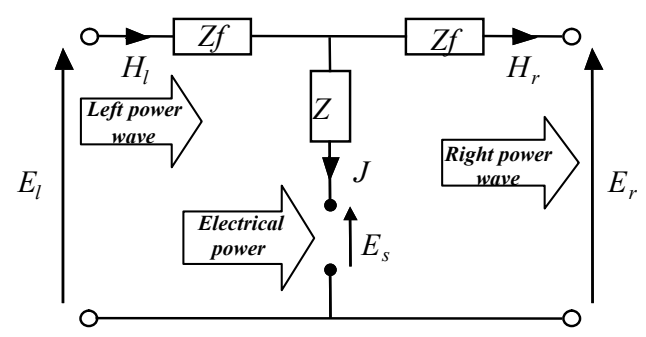

Fig. 3. Equivalent physical circuit associated to a conductive plate

Input-output symmetry of this circuit results of corresponding symmetry of the layer and the two included impedances are given by (4).

$$
\begin{aligned}
& Z_{f 1}=Z_{c} \frac{1-A}{1+A}=j Z_{c} \tan (\varphi / 2) \\
& Z_{f 2}=Z_{c} \frac{2 A}{1-A^{2}}=-j Z_{c} / \sin \varphi
\end{aligned}
$$

A simple change of circuit shown in figure 3 brings electrical variables linked to the external circuit into light. Indeed, replacing $J$ by $I$ (total current supplied by the source) needs only a multiplication by the plate width $b$. In the same way, electric fields can be replaced by voltage owing to a multiplication by the plate length $c$. So, after multiplication of all currents by $b$ and all electrical fields by $c$, circuit of figure 4 is consistent with that of figure 3 if $Z_{f 1}$ and $Z_{f 2}$ are both multiplied by $c / b$ to gives $Z_{1}$ and $Z_{2}(5)$.

$$
\begin{aligned}
& Z_{1}=j Z_{c} \frac{c}{b} \tan (\varphi / 2) \\
& Z_{2}=-j Z_{c} \frac{c}{b} \frac{1}{\sin \varphi}
\end{aligned}
$$

At last, if a conducting layer is made of $N$ strands connected in series, input current source must be multiplied by $N$ to give the total current going through the layer while input voltage must be divided by $N$ to obtain the voltage across one strand. This is easily done by introducing an $\mathrm{N}$-ratio coupler (Fig. 4). In practice, we keep this coupler in the circuit, even when its ratio is 1 , to insure electrical insulation of each electrical input.

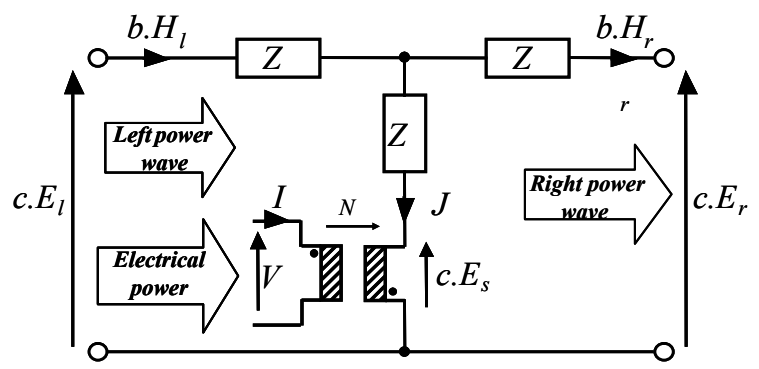

Fig. 4. Equivalent electrical circuit associated to a conductive plate 
To understand the meaning of impedance $Z_{1}$ and $Z_{2}$, it is in interesting to look at their low frequency limits (6).

$$
\begin{aligned}
& Z_{1}=j \sqrt{\frac{\bar{\mu}}{\bar{\varepsilon}}} \frac{c}{b} \frac{1}{2} \omega \sqrt{\bar{\mu} \bar{\varepsilon}} a=j \bar{\mu} \frac{c}{b} \frac{a}{2} \omega \\
& Z_{2}=-j \sqrt{\frac{\bar{\mu}}{\bar{\varepsilon}}} \frac{c}{b} \frac{1}{\omega \sqrt{\overline{\mu \varepsilon} a}}=\frac{1}{j \bar{\varepsilon} \omega} \frac{c}{a b}
\end{aligned}
$$

In the low frequency side, $Z_{1}$ is the inductance given by a reluctance of permeability $\bar{\mu}$ (equal to $\mu_{0}$ for copper), length $b$ and section $c a / 2$. It looks like the leakage inductance introduced by half the thickness of the plate (magnetic field is parallel to dimension b). In the same range of frequency, $Z_{2}$ is the resistance of the plate because, in low frequency, second term of (1) dominates the value of $\bar{\varepsilon}$, so first fraction of $Z_{2}$ in (6) is the reverse of $\sigma$. We must admit that, despite their complicated expressions that account for their frequency variations, these impedances appear quite natural.

For a non supplied plate (insulator, magnetic material) vertical branch of circuit of fig. 4 is not relevant and circuit reduces to unique impedance equal to $2 Z_{1}$. So, according to this, an insulating layer of thickness a, is introduced, in the electrical circuit, as an inductance $l_{a}$ given by (7).

$$
l_{a}=\mu_{0} \frac{a c}{b}
$$

Same reasoning leads to represent a magnetic layer by an inductance $l_{m}$ (having losses) given by (8). Because theory suppose magnetic field is mull outside the core [9], electric field is null on the external surface of this plate and one terminal of related impedance is at the reference voltage. So, it is useful to remind these impedances supply the return path for all currents injected as shown in Fig. 5.

$$
l_{m}=\bar{\mu} \frac{a c}{b}
$$

This value is tightly linked to the al factor (inductance per square turn) given by core manufacturers. Generally it equals twice this value because two plates are needed to represent the whole magnetic core. Despite core permeability is given on a wide frequency range, only low frequency value of the inductance per square turn is commonly specified. Its value in H.F. is calculated as follows (9).

$$
a l(f)=\operatorname{al}(0) \frac{\overline{\mu(f)}}{\overline{\mu(0)}}
$$

\section{Representation of a Plate Using Passive Impedances}

Frequency dependant impedances are not easily taken into account by circuit simulation software and using them for time domain simulation is quite impossible. For this reason, we now look for lumped element circuits that can accurately replace circuits found above.

Because permeability of an insulating layer is equal to that of vacuum on the whole frequency range, its representation only require a constant inductance. Capacitive aspects will be studied in II- $E$.

Representation of a magnetic layer must account for all aspects of permeability variation with frequency, including losses. The needed impedance is designed, in

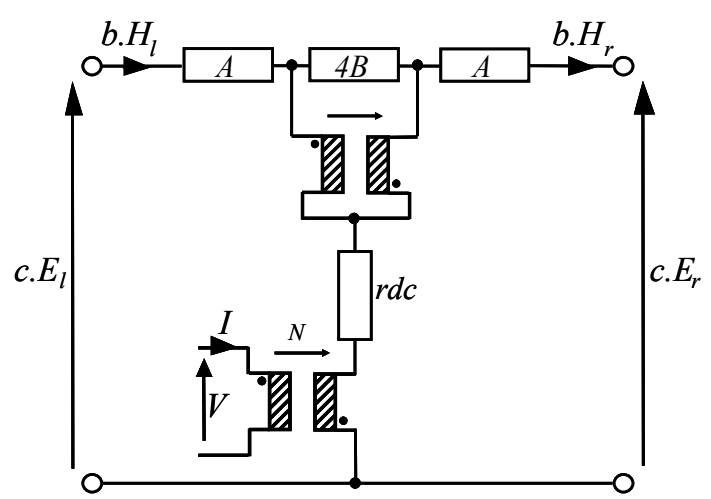

Fig. 5. Final equivalent 1 circuit associated to a conductive plate

each case, by looking at manufacturer data. An example will be given in section III. The roughest approximation is given by an inductance paralleled by a resistance often called "iron losses resistor".

Conductive plate is more complicated to represent (several impedances to design) and, due to eddy current effects, its impedances begin varying at rather low frequency so these variations can't be neglected at all.

In a first try, we kept the resistor $r d c$ found as the low frequency limit of $Z_{2}(6)$ in series and we remarked that the remaining part of $Z_{2}$ was negative. That led us to introduce a second coupler in the circuit (Fig. 5) to naturally account for this negative part on the mutual impedance. Identification of circuits of figures 4 and 5 gives expressions of $A$ and $B$.

$$
\begin{aligned}
& A=Z_{1}+2 Z_{2}-2 r d c \text { and } B=r d c-Z_{2} \\
& \text { where: } \quad r d c=\frac{1}{\sigma} \frac{c}{a b}
\end{aligned}
$$

Frequency dependence of these impedances (11) is clarified by returning to (2), (3), (5) and (6).

$$
\begin{aligned}
& A=r d c 2\left(\frac{(1-j) \sqrt{\frac{f}{f_{s}}}}{\tan \left((1-j) \sqrt{\frac{f}{f_{s}}}\right)}-1\right) \\
& B=r d c\left(1-\frac{2(1-j) \sqrt{\frac{f}{f_{s}}}}{\sin \left(2(1-j) \sqrt{\frac{f}{f_{s}}}\right)}\right)
\end{aligned}
$$

In these expressions, $f_{S}$ (we call skin frequency) is the frequency for which skin depth $\delta(12)$ equals half the plate thickness $a$ (12). Its value is close to $1 \mathrm{MHz}$ for a $125 \mu \mathrm{m}$ thick copper layer.

$$
\delta=\sqrt{\frac{2}{\omega \sigma \mu_{0}}} \Rightarrow f_{s}=\frac{4}{a^{2} \pi \sigma \mu_{0}}
$$

Owing to this change, involved impedances $A$ and $B$ have positive real parts and their frequency behaviors are consistent with that of passive impedances (Fig 6). Impedances $B$ shows strongly damped resonances that are related to propagation through the plate. Fig. 7 shows that these resonances appear when thickness is a half integer multiple of the wavelength $\lambda(=2 \pi \delta$ in this material). 

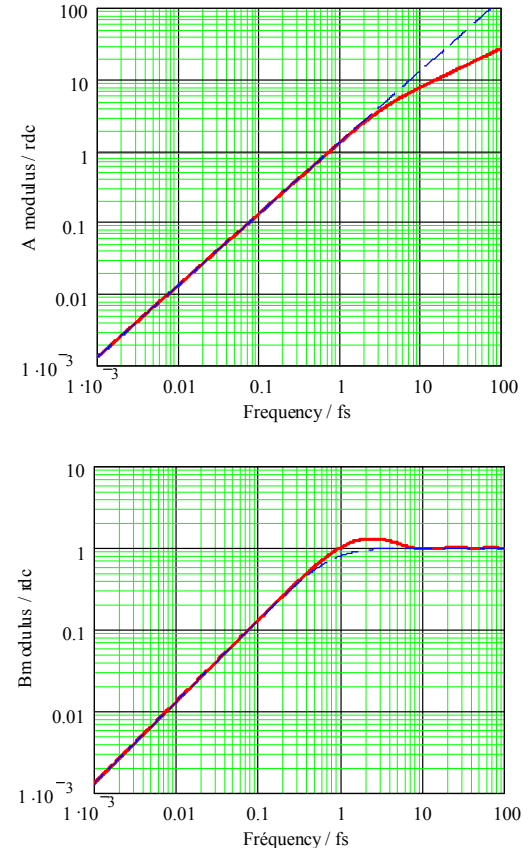

Fig. 6. Variation of impedances modulus ( $A$ and $B$ ) vs. frequency

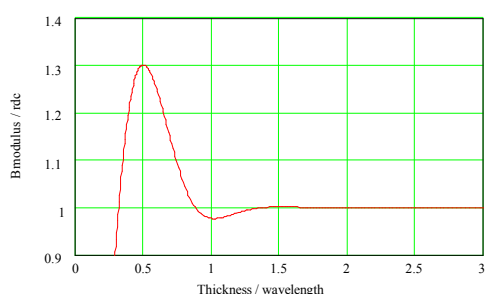

Fig. 7. Modulus of $B$ vs. thickness / wavelength

Both impedances look inductive in the low frequency side. For $B$, a $L p-R p$ approximation suits pretty well (dotted line) but HF asymptote of $A$ is of half order so representation by a finite number of components will have, necessarily, a bounded frequency range of validity.

\section{Lumped Element Representation of Impedances}

Now we begin looking for lumped element equivalent circuits for $A$ and $B$, expecting modulus inaccuracy to be under $1 \%$ up till, at least, $30 f s$.

To reach this result for $B$, we use Pade approximations [10] three times. We first develop admittance in Mac Laurin series of the frequency and we keep the first complex term (which corresponds to a resistor paralleled by an inductor). Then, the remaining part of the admittance is inverted and the result is also developed in Mac Laurin series. We keep the first complex term that gives a resistor and a capacitor in series. One more time, developing remaining part gives an inductor paralleled by a resistor.

At this point, obtained equivalent circuit (Fig. 8) shows impedance variation very close $(+2.15 \%,-1.2 \%)$ to that

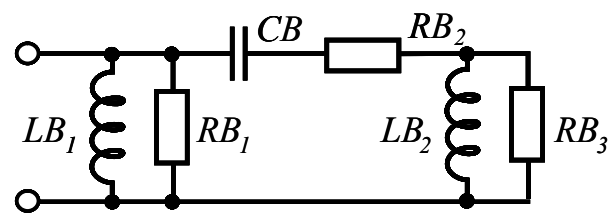

Fig. 8. Equivalent circuit for impedance $B$
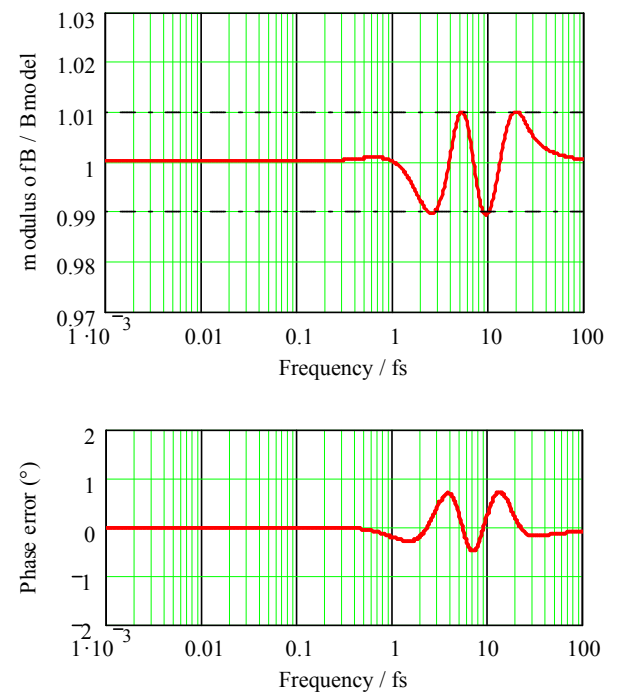

Fig. 9. Accuracy of representation of $B$ by lumped elements

of original circuit, over the full frequency range. A slight adjustment of component values suffices to reach targeted accuracy (Fig. 9). Component expressions are grouped in table 1.

TABLE 1. ExPREsSions of Component VAlues for $B$. BEFORE (2 FiRST LiNES) AND AFTER TUNING

\begin{tabular}{|l|l|l|}
\hline Putting: $\quad r d c=\frac{1}{\sigma} \frac{c}{a b} \quad l d c=\mu_{0} \frac{a c}{b} \quad c d c=\mu_{0} \sigma \frac{a^{3} b}{c}$ \\
\hline$L B_{1}=\frac{1}{6} l d c$ & $C B=\frac{11}{1400} c d c$ & $L B_{2}=\frac{25}{1782} l d c$ \\
\hline$R B_{1}=\frac{10}{7} r d c$ & $R B_{2}=\frac{2380}{1089} r d c$ & $R B_{3}=\frac{7865}{4869} r d c$ \\
\hline$L B_{1}:$ unchanged & $C B: \times .942$ & $L B_{2}: \times 2.44$ \\
\hline$R B_{1}:$ unchanged & $R B_{2}: \times .761$ & $R B_{3}: \times 1.303$ \\
\hline
\end{tabular}

Now, we focus on impedance $A$. On the low frequency side, this impedance behaves as an inductance equal to $l d c / 6$. Beyond $.77 f s$ this approximation is not accurate enough (fig. 6). To extend the frequency range of validity, we alternatively divide and multiply this initial impedance, by a degree 1 polynomial as shown by (13). We note this impedance $\mathrm{Am}$ ( $m$ like model).

$$
A m=j \frac{1}{6} l d c 2 \pi f \frac{1+j \frac{f}{f c_{2}}}{1+j \frac{f}{f c_{1}}} \frac{1}{1+j \frac{f}{f c_{3}}} \cdots
$$

In practice, to adjust parameters $f_{c_{1}}$ to $f_{c_{3}}$, we introduce related terms one by one. For one extra term, we adjust $f c_{1}$ to keep the modulus of the ratio: $A m / A$, in the allowed interval (.99 to 1.01) up till a frequency as high as possible. Then, we add the term that depends on $f c_{2}$ and, starting from the previous value of $f_{c_{1}}$ we adjust $f_{c_{1}}$ and $f_{c_{2}}$ to extend the frequency limit of accuracy as high as possible. We find $f_{c_{3}}$ using the same method so parameters of expression (13) are now all known.

To finish that process, we express Am/ldcs as a function of $s=j 2 \pi f$ and we expand this rational fraction of 


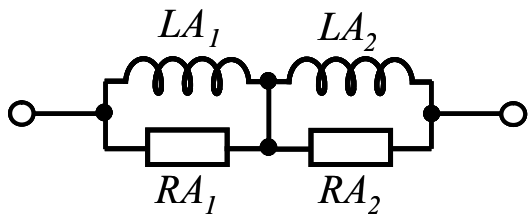

Fig. 10. Equivalent circuit for impedance $A$

$s$ in simple elements. Each of them is easily identified to a $L p-R p$ cell. Corresponding circuit is represented in figure 10 and component values are given in table 2 . Accuracy of modulus and phase is checked in figure 11. It reaches our target from d.c. up till $46.2 \mathrm{fs}$.

TABLE 2. EXPressions of COMPONENT VALues for $A$.

\begin{tabular}{|l|l|}
\hline$L A_{1}=.115 \mathrm{ldc}$ & $L A_{2}=51.410^{-3} \mathrm{ldc}$ \\
\hline$R A_{1}=4.93 \mathrm{rdc}$ & $R A_{2}=15.92 \mathrm{rdc}$ \\
\hline
\end{tabular}

As indicated before, validity of equivalent circuit for $A$ has, contrary to $B$, a high frequency limit. However, let us remind that, for a $125 \mu \mathrm{m}$ thick copper layer, $f_{s}$ is located at about $1 \mathrm{MHz}$ so, with usual thickness used in planar technology, we can be confident in proposed circuits up till, at least, $20 \mathrm{MHz}$. At this frequency, we have no manufacturer data regarding our magnetic core....

\section{E. Representation of Capacitive Behavior}

Theory presented above relies on the propagation of plane waves normally to all plates. For this reason, the only component of electric field taken into account is parallel to the plates. Obviously, this component is not the most significant for the study of capacitive properties. To compensate this lack, another model is now joined to former one.

In studied transformers, metallic plates of length $\mathrm{c}$ and width $b$ are separated by dielectric material of thickness $a$. This suggests introducing a simple capacitor between each pair of metallic plates but, contrary to plane capacitors, metallic plates are not equipotential. Indeed, electrostatic field in these regions depends on 3 independent voltages.
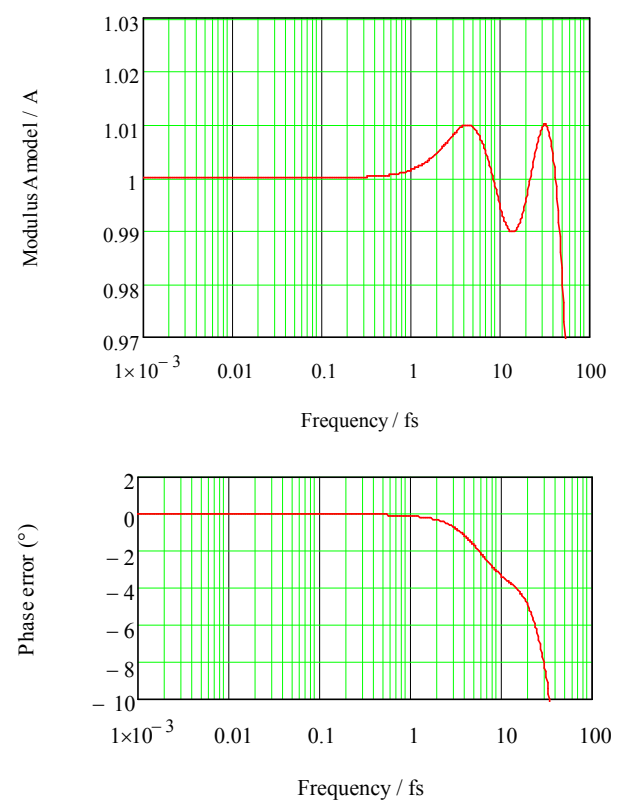

Fig. 11. Accuracy of representation of $A$ by lumped elements

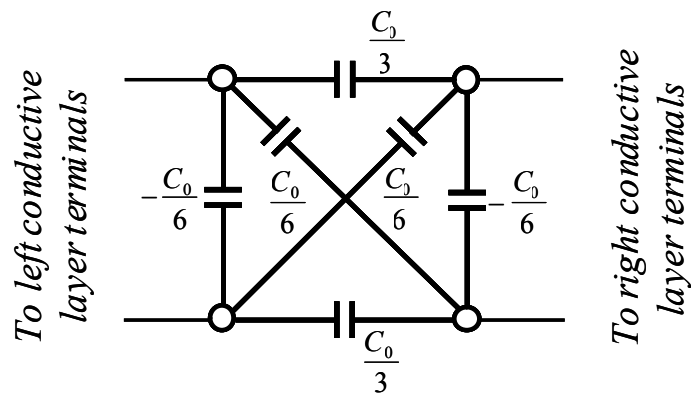

Fig. 12. Equivalent circuit for interlayer capacitances

If we suppose, for each metallic layer (= one turn), that potential varies linearly when going from one terminal to the opposite, electric field in each dielectric layer is easily computed and, according to [11], 6 capacitors (Fig. 12), placed between the four terminals of the two surrounding conductive layers, suit to fully account for electrostatic properties.

In figure $12, C_{0}$ equals the capacitance of a plane capacitor $(=\varepsilon b c / a)$. This value is seen between the two layers when they both have a null voltage across them. Two negatives values appear in this circuit but all directly measurable capacitances are positive.

\section{COMPARISON WITH MEASUREMNTS}

\section{A. Component Short Description}

Tested component is that shown in figure 1. It is a 3windings device made of 38 copper layers among which are 14 screens. All these layers are $105 \mu \mathrm{m}$ thick and they are separated by same insulating material of various thickness. Magnetic core comes from TDK. It is a PQ59 core that has been machined as described below. It is made of PC40 ferrite.

\section{B. Circuit Introduced in PSpice}

To begin, every copper layer is represented by circuits shown in figures 5, 9 and 10. For a thickness of $105 \mu \mathrm{m}$, skin frequency $f_{S}$ equals $1.5 \mathrm{MHz}$. Looking at figures 9 and 11 , one can know the expected accuracy of this representation.

Used insulating material is a composite one, the relative permittivity of which is close to 4.5. All insulating layer, including electrostatic screens, have been represented according to figure 12 . These circuits are connected to floating sides of input couplers belonging to adjacent conductive layers.

Representation of ferrite layers needs some extra explanations. First, PQ 59 core has been grinded in order to first reduce its thickness of $5 \mathrm{~mm}$, second, introduce an $.37 \mathrm{~mm}$ thick air gap air gap. This reduces inductance per square turn $\mathrm{Al}$ to $\mathrm{Alm}$ as follows. Initial length of mean field line equals $80 \mathrm{~mm}$ and $10.37 \mathrm{~mm}$ is subtracted of this length so:

$$
\begin{aligned}
& A l m=A l \frac{80}{69.63} \text { where } A l=12.81 \mu H / N^{2} \\
& A l m=14.72 \mu H / N^{2}
\end{aligned}
$$

In practice, this inductance is not pure, it slightly varies with frequency and it introduces losses. This is directly linked to complex permeability variation (Fig. 13). 


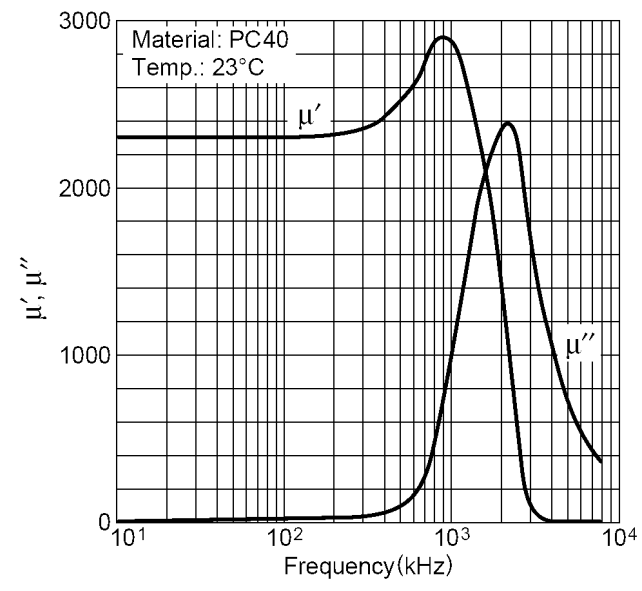

Fig. 13. Permeability vs. frequency, as given by TDK

Accounting for this is simple (15) because inductance is proportional to permeability.

$$
\operatorname{Alm}(f)=\operatorname{Alm} \frac{\mu r(f)}{\mu r(0)}
$$

This modification leads to replace the pure inductance by a four component equivalent circuit (Fig. 14). This simple model agrees with manufacturer data with less than $10 \%$ of discrepancy from d.c. to $8 \mathrm{MHz}$.

To end, we now focus on air gap. Knowing its thickness e and the surface $S m$ of the magnetic core, calculating its reluctance is easy. Related inductance (the reverse of the reluctance) is a pure inductance given by (16) which intervenes in parallel with impedance linked to ferrite as usual for reluctances in series.

$$
L a=\mu_{0} \frac{S m}{e}=1.56 \mu H
$$

Due to this inductance in parallel, circuit of Fig. 14 must be used with an inductance value equal to $1.41 \mu \mathrm{H}$.

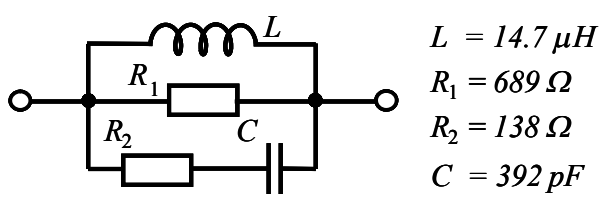

Fig. 14. Impedance per square turn: lumped element equivalent circuit

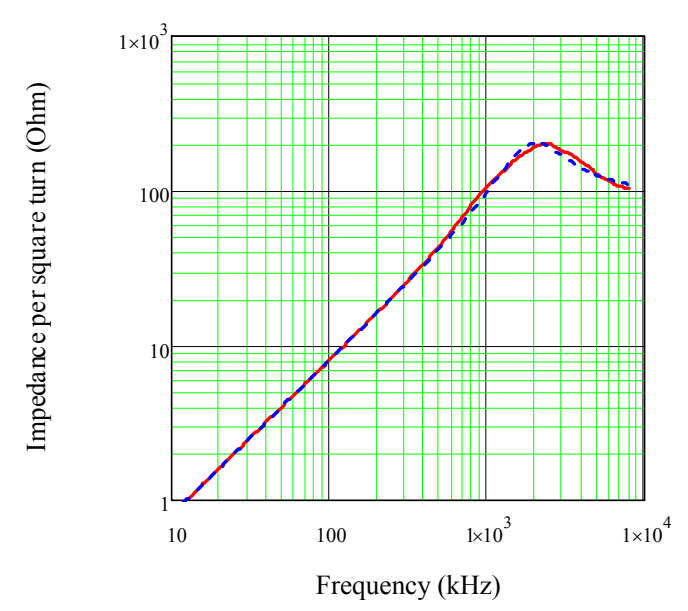

Fig. 15. Impedance per square turn: Solid line: manufacturer data, dotted line: model of figure 14
We are now able to complete the introduction of our circuit in PSpice. Because elements are numerous, this is a little bit long but all metallic layers being identical, that piece of circuit is repeated 38 times. Final circuit includes 479 resitors, 542 inductors, 262 capacitors, 76 couplers... for a total of about 1500 components.

As a first step, we use it with sinusoidal excitation in order to compare the results with impedances measured habitually to identify such a component. Despite this transformer owns three windings, to clarify this presentation, we focus on a pair of them. Other one and internal screens are kept floating.

Fig. 16 shows measured and simulated results. $Z p 0$ and $Z p c c$ are impedances seen from primary side with, respectively, secondary open and shorted. In the same manner, $Z s 0$ and $Z s c c$ are seen from the secondary side.

To reach a so good agreement, only one component has been added to the circuit described above: a weak inductance $(6 n H)$ has been added in series with secondary winding. Indeed, a first simulation showed good agreement for many things but simulated short circuit impedances were about 10 times too weak. After a short moment, it appeared to us that, close to secondary terminals, the structure of our transformer doesn't respect our model requirements.

Large conductors with screws seen on figure 1 are connected to circular turn by parallel conductors which
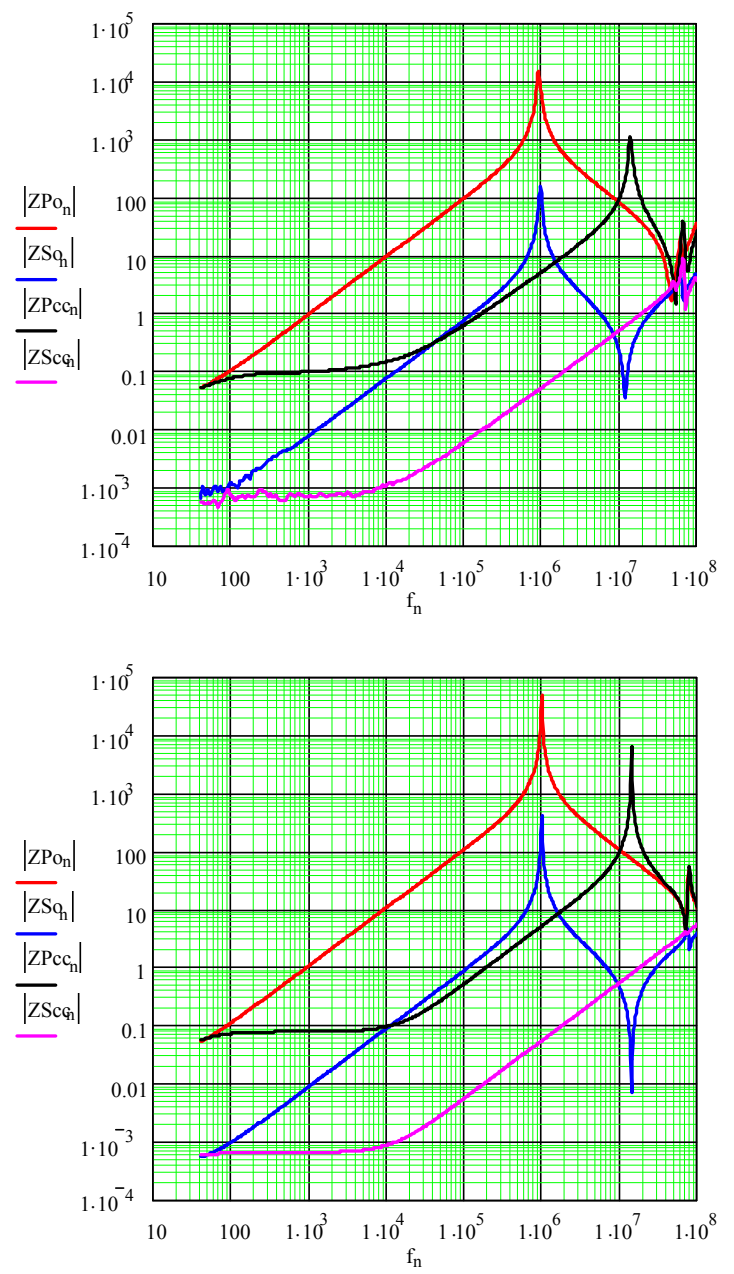

Fig. 16. Measured and simulated impedances curves 

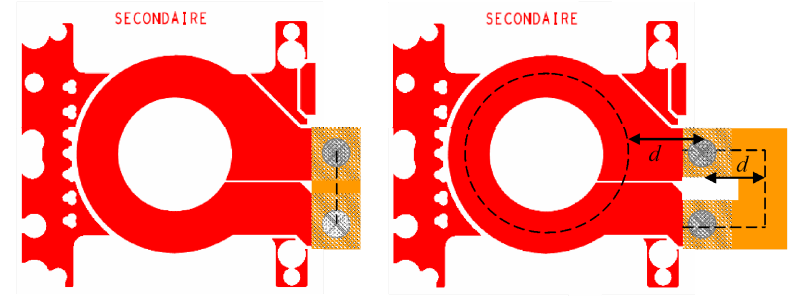

Fig. 17. Two different short-circuits for output impedance evaluation

don't face each over. So, we supposed this pair of parallel conductors, $2 \mathrm{~cm}$ long, to be responsible for a small $(6 \mathrm{nH})$ but significant inductance, not taken into account by our approach.

To check that hypothesis, we replaced our initial shortcircuit by a U-shaped one (Fig. 17) in order to prolong incriminated conductors by $2 \mathrm{~cm}$. This, of course, increased the inductance seen from primary side and we deduced the inductance experimentally added to the secondary side. Because conductors responsible for this increase had about the same dimensions that those initially present in the transformer and which are not taken into account, we thought found inductance value also characterized to the guilty part of the transformer. As a conclusion, we introduced an inductance of the same value $(6 n H)$ in series with the secondary winding of our equivalent circuit. Result of this is the agreement seen in Fig. 16.

Before concluding, we wish to share our opinion about the relative places of equivalent circuit introduced in this paper and global circuit deduced from measurements. As mentioned before, equivalent circuit studied in this paper can include more than two thousand elements. This can be heavy to run time domain simulation of a complete circuit. Fortunately, there is an alternate way to proceed. Using curves like those of figure 16 , we have developed methods $[3,4,5]$ that lead to a global equivalent circuit which suffices to forecast all the external behavior of a transformer, even if submitted to complex waveforms. This global circuit only includes a small number of components: generally twenty to fifty. After the simulation of the entire circuit with the global equivalent circuit, all input waveforms of the transformer are known. If necessary, these waveforms can be applied to the transformer alone, depicted by its detailed equivalent circuit, in order to reach fine internal behavior.

\section{CONCLUSION}

A new way of forecasting electrical behavior of planar transformer has been presented. It applies for transformers having simple but common geometries. Assuming they only have a circuit simulator like PSpice, engineers can now deduce, from physical and geometrical description of their component, its complete electrical behavior, from d.c. to several tens of $\mathrm{MHz}$.

Linear H.F. properties of the core, eddy current effects in conductors and stray capacitive effects are taken into account. Owing to the use of a circuit simulator, after the description of the component, the simulation lasts a few seconds. Notice that, with a fem simulator, these problems are very difficult to solve because, at $10 \mathrm{MHz}$, skin depth equals $20 \mu \mathrm{m}$ so meshing must be very fine.

Because our equivalent circuit only includes lumped elements, it suits for harmonic studies and complex waveforms as well. Electrical description of the device is complete enough to forecast the results of measurements generally carried out to characterize these components. In a second step, a reduced equivalent circuit, which only includes 20 to 50 lumped elements, can be deduced from these simulated measurements.

Inter-winding impedances, current sharing between parallel turns [11,12], impact of electrical screens on behavior are, among many others, properties that can be studied this way for a fixed topology transformer. More interesting is the possibility of quickly compare several designs for a given component. Following this approach, comparing several interleaving of windings, checking impact of a screen introduction or of a dielectric thickness is done very fast.

\section{ACKNOWLEDGMENT}

We want to warmly thank Thales Airborne Systems for their long term interest in our work and their financial support.

\section{REFERENCES}

[1] X. Margueron, J-P. Keradec, "Design of equivalent circuits and characterization strategy for n-input coupled inductors", IEEE Trans. on Industry Applications, Jan.-Feb. 2007, vol. 43, no. 1, pp. 14-22.

[2] PSpice information at http://www.cadence.com/orcad/index.html.

[3] B. Cogitore, J-P. Keradec, J. Barbaroux, "Two winding ferrite core transformer: an experimental method to obtain a wide frequency range equivalent circuit". IEEE Transactions on Instrumentation and Measurements, April 1994, vol. 43, no. 2, pp. 364-371.

[4] X. Margueron, J-P. Keradec, "Identifying the magnetic part of the equivalent circuit of n-winding transformer", IEEE Transactions on Instrumentation and Measurements, Feb. 2007, vol. 56, no. 1, pp. 146-152.

[5] J.P. Keradec, Identification expérimentale progressive des circuits équivalents de transformateurs HF. Les techniques de l'ingénieur (F), dossier D3059, to be published in Nov. 2008.

[6] A. Schellmanns, P. Fouassier, J. P. Keradec, J. L. Schanen, "Equivalent circuit for transformers based on one-dimensional propagation: accounting for multilayer structure of windings and ferrite losses", IEEE Transactions on Magnetics, Sep. 2000, vol. 36 , no. 5 , pp. 3778-3784.

[7] L. Heinemann, "Modelling and design of high frequency planar transformers",. PESC '95 Record, vol.2, pp. 651 - 657.

[8] P. Lorain, D. Corson, "Electromagnetic fields and waves", second edition, W. H. Freeman and company, San Francisco (CA), USA, 1962, 1990.

[9] J. P. Keradec, B. Cogitore, F. Blache, "Power transfer in a two winding transformer: from $1 \mathrm{D}$ propagation to an equivalent circuit". IEEE Transactions on Magnetics, Jan. 1996, vol. 32, no. 1, pp. 274-280.

[10] Brezinski, C.; and Redivo Zaglia, M. Extrapolation Methods. Theory and Practice. North-Holland, 1991.

[11] E. Laveuve, J. P. Keradec, M. Bensoam, "Electrostatic of wound components: analytical results, simulation and experimental validation of the parasitic capacitance", IEEE Industrial Application Society, Dearborn (Mi, USA), 28 Sept. - 4 Oct. 1991, Proc. vol. 2, pp. 1469-1475.

[12] X. Margueron, J.-P. Keradec, A. Besri : "Current sharing between parallel turns of a planar transformer: prediction and improvement using a circuit simulation software", IEEE Industrial Application Society 2007, New Orleans (LA, USA), 23-27 Sept. 2007.

[13] R. Prieto, J-A Cobos, O. Garcia, P. Alou, J. Uceda, "Using parallel windings in planar magnetic components", Power Electronics Specialists Conference, 2001, Vancouver, BC, Canada, Proc vol. 4, pp. 2055-2060. 